\title{
RESEARCH
}

\section{DOES AGING AFFECT BENIGN PAROXYSMAL POSITIONAL VERTIGO TREATMENT?}

Turkish Journal of Geriatrics

DOI: 10.31086/tjgeri.2021.211

2021; 24(2): 159-164

- Mine BAYDAN ARAN ${ }^{1}$

- Özge Selen AVCI CAN ${ }^{1}$

- Zehra AYDOĞAN ${ }^{1}$

- Zahide ÇILER BÜYÜKATALAY²

CORRESPONDANCE

${ }^{1}$ Mine BAYDAN ARAN

Ankara University, Department of Audiology,

Ankara, Turkey

Phone: +905312929012

e-mail: mine.baydan@gmail.com

Received: Apr 14, 2021

Accepted: May 21, 2021

${ }^{1}$ Ankara University, Department of Audiology, Ankara, Turkey

${ }^{2}$ Ankara Universiy, Department of Ear, Nose and Throat, Ankara, Turkey

\begin{abstract}
Introduction: Benign Paroxysmal Positional Vertigo is seen in all age groups; however, its incidence increases with age. In addition to many factors affecting its treatment, aging is also thought to have an effect on treatment. The aim of this study is to investigate the relationship between the number of maneuvers applied in treatment and patient's age, gender and the affected
\end{abstract} canal.

Materials and Method: In total 290 subjects, between 18 to 87 years were included in the study. The positional tests were performed and the appropriate maneuver was performed based on affected canal. Following third day the maneuver positional tests were repeated. If the symptoms and / or nystagmus continued, maneuver was performed again.

Results: It was found that older adults require higher number of maneuvers to recover $(p<0.05, r=0.33$ ). In addition, comorbidities such as hypertension and migraine were observed more frequently in older adults compared to the other groups.

Conclusion: Changes and comorbidities caused by aging render treatment process far and difficult in the elderly.

Keywords: Aging; Vertigo; Comorbidity; Vestibular Diseases 


\section{INTRODUCTION}

Benign Paroxysmal Positional Vertigo (BPPV) is a peripheral vestibular system disorder characterized by a sudden and short-term vertigo, dizziness, nausea, and positional nystagmus caused by the movement and/or placement of the head relative to the affected semicircular canal. Although BPPV is benign, it affects patients' daily life activities and can lead to morbidity or disability due to falls (1-3).

BPPV can be seen in all age groups, however it has been reported that the mean age of onset is in the fourth and fifth decades and its incidence increases with age $(3,4)$. Additionally, as it has been observed, BPPV is 7 times more likely to be seen in patients over 60 years of age than in the 18-39 age groups (5). It has been stated that morphological damage to otolith organs and decrease in the number of hair cells in these organs due to aging may cause BPPV $(5,6)$.

In the treatment of BPPV, appropriate maneuvers are applied based on the affected semicircular canal (2). However, while only one maneuver can be sufficient in some BPPV cases, in others, repetitive maneuvers may be required (7). There are many factors affecting the effectiveness of the treatment, among which are age, gender, affected ear and/or canal and comorbidities $(8,9)$. The aim of this study is to investigate the relationship between the number of maneuvers applied in BPPV treatment and patient's age, gender and the affected canal.

\section{MATERIALS AND METHOD}

The study protocol was approved by Ankara University Faculty of Medicine Non-Interventional Clinical Research Ethics Committee (No: i7 - 440 - 20). According to the national legislations and institutional rules and procedures, all patients are obliged to sign an informed consent form approving analysis of their clinical records, and publication of their anonymous data.

After reviewing the medical records of patients admitted to Ankara University School of Medicine İbn-i Sina Hospital, Otorhinolaryngology Department, Hearing, Speaking and Balance Disorders Center between September 2018 and June 2020 with dizziness/balance disorder, patients over 18 years of age and diagnosed with BPPV were included in this study. Subjects with neurological disorders and/or with multicanal involvement BPPV were excluded. The age, gender, affected canal, number of maneuvers and comorbidities of BPPV subjects were recorded.

The subjects were divided into three age-based groups: "Group 1" consists of subjects who are 1844 years of age; "Group 2," of subjects who are 4564 years of age, and "Group 3" consists of subjects over 64 years of age.

Each subject was initially examined with videonystagmography (VNG) as a standard procedure. Eye movements were recorded using VisualEyes VNG (Chatham, IL, USA). Saccadic and smooth pursuit eye movements, optokinetic, gaze, and spontaneous nystagmus were also recorded. Analysis on saccades was conducted for accuracy, velocity, and latency, whereas smooth pursuit and optokinetic tracking were analyzed for symmetry and gain.

The positional tests were performed with VNG googles. The Dix Hallpike test was employed for diagnosis in posterior and anterior semicircular canal BPPV, and Roll test was used in the diagnosis of lateral semicircular canal BPPV. Once the affected canal was identified, an appropriate maneuver, chosen in accordance with the BPPV's pathophysiology (canalolithiasis, cupulolithiasis) and affected canal, was performed in the same session (one maneuver per session). Following the maneuver, the patient was recommended to avoid sudden head movements and to keep their head in an elevated position while sleeping. Three days after the maneuver, the test was repeated. In cases where symptoms and nystagmus continued, maneuver was performed again. 


\section{STATISTICAL ANALYSIS}

Data were analyzed using SPSS version 26.0. Quantitative data were described as mean $\pm S D(X \pm S D)$, and qualitative data were described in percentage values. The normality of data was evaluated with visual (histogram and stem-leaf plots) and analytic (Kolmogorov-Smirnov/Shapiro-Wilk tests) methods. The differences between groups were analyzed with Kruskall-Wallis test, and any differences were to be found, two-way comparisons were held by Mann Whitney $U$ test (the comparisons were between group 1-2, 1-3, 2-3). Additionally, the relationship between age and maneuver count was investigated with Pearson correlation analysis.

\section{RESULTS}

Forty-two of 332 patients diagnosed with BPPV were excluded due to multi-canal BPPV and a total of 290 patients were included in the study. Group 1 consisted of 57 females and 20 males with mean age of $35.32 \pm 7.33$ years. Group 2 consisted of 95 females and 26 males with mean age of $55.29 \pm 5.49$ years. Group 3 consisted of 63 females and 31 males with mean age of $73.08 \pm 5.85$ years.

In an overall assessment, the most affected canal was observed to be the right posterior canal (38.62\%), followed by the left posterior canal (29.65 $\%)$, lateral canals $(21.72 \%)$, anterior canals $(10.68 \%)$, respectively.

An evaluation of the medical history of participants provided the following data: In Group 1, 3.89\% of participants have hypertension; $5.19 \%$ have migraine, and $10.38 \%$ are afflicted by other diseases. In Group 2 hypertension rate is $29.75 \%$, diabetes is $21.48 \%$, and $23.14 \%$ of the participants had other diseases. In Group 3 the incidence of these factors was $59.57 \%, 32.97 \%$, and $24.46 \%$, respectively.

The distribution of the average number of maneuvers by groups is given in Table 1. Comparison of the groups provided in Table 2 reveals that statistically, average number of maneuvers in
Group 3 is significantly higher than in Groups 1 and $2(p<0.001)$.

Table 1. Average Number of Maneuvers by Groups

\begin{tabular}{|l|c|c|c|}
\hline \multicolumn{1}{|c|}{ Maneuver } & Group 1 & Group 2 & Group 3 \\
\hline Minimum & 1 & 1 & 1 \\
\hline Maximum & 5 & 5 & 9 \\
\hline Mean-SD & $1.79 \pm 1.04$ & $1.80 \pm 0.89$ & $2.89 \pm 1.66$ \\
\hline
\end{tabular}

*SD: Standard Deviation

Table 2. Comparisons of the Average Number of Maneuvers Between Groups

\begin{tabular}{|c|c|}
\hline \multicolumn{1}{|c|}{ Groups } & p \\
\hline Group 1 - Group 2 & 0.504 \\
\hline Group 1 - Group 3 & $0.00001^{*}$ \\
\hline Group 2 - Group 3 & $0.00003^{*}$ \\
\hline
\end{tabular}

${ }^{*} \mathrm{p}<0.05$, Mann Whitney U Test

\section{DISCUSSION}

The primary purpose of the present study was to investigate whether aging affects the maneuver rehabilitation process of BPPV. Although some studies state that the number of maneuvers required is independent of age $(10,11)$ the Group 3 results in this study demonstrate that older BPPV patients need more repetitions, and this is thought to be caused by, for example, reduced effectiveness due to waist and neck restrictions from orthopedic problems, comorbidities, or not following post-treatment movement recommendations.

The current BPPV literature suggests many reasons for requiring repeated maneuvers, the most 
common of which are comorbidities such as hypertension and diabetes $(4,9,11,12)$. In this study, the group of older participants indicated a hypertension ratio of $59.75 \%$ and needed more maneuvers, and this is compatible with these existing reports. Relatedly, it has been suggested that the increased prevalence and prolonged treatment of BPPV due to age may be caused by separation of the otoconia from the macula or degenerative changes in the utricle due to chronic ischemia $(12,13)$.

Orthopedic problems are also considered to be a reason for the need for multiple maneuvers (14, 15). For example, Balatsouras et al., state that issues such as limited cervical motion, kyphosis, limited range of motion, or low back and neck pain can affect the effectiveness of repositioning maneuvers in BPPV treatment (7). Likewise, it is our experience that orthopedic restrictions have a negative impact on the effectiveness of maneuver treatment, and the use of repositioning chairs in these cases can be beneficial.

In accordance with the literature, the present study indicates that the right posterior is the most commonly affected canal in BPPV diagnosis, followed by the left posterior (16-18). However, there was no significant relationship between the affected canal and the number of maneuvers required for successful treatment.

BPPV in the elderly differs from that in younger patients in terms of pathogenesis, diagnosis, and treatment, and older patients also demonstrate higher prevalence, are less responsive to treatment, and show a greater tendency to recurrence $(12,13)$. However, the number of participants in the younger Group 2 was higher than in Group 3 in this study, although this inconsistency in age profile can be attributed to the difficulty of obtaining accurate anamnesis from older patients, misdiagnosis due to similar symptoms of comorbidities, and/or vascular and orthopedic problems and their consequent referral to different departments.

Additionally, $74.13 \%$ of the participants in the current study were women, and this majority finding is consistent with previous investigations (19-21), some of which have attributed BPPV to hormonal fluctuations, menopause, and/or osteoporosis (22, 23). One associated limitation of this study is the lack of specific information about osteoporosis and the menopause, and so it is recommended that all comorbidities are examined in greater detail in future studies.

Falls are common in the geriatric population, particularly in those with chronic debilitating conditions such as osteoporosis or neurological limitations (24). In such cases, BPPV is an increased risk factor for falls, and its immediate treatment is therefore crucial. Therefore, the strength of this study is that it draws attention to the factors affecting the BPPV rehabilitation process in geriatric individuals. It was aimed to raise awareness that comorbidities prolong the process and this situation increases the risk of falling.

In conclusion, this study demonstrates that changes and comorbidities due to aging render maneuver treatment for BPPV more difficult in elderly populations. Nevertheless, failure to treat BPPV can increase the risk of falls, negatively affects quality of life, and increases the burden of healthcare costs $(9,16)$. 


\section{REFERENCES}

1. You P, Instrum R, Parnes L. Benign paroxysmal positional vertigo. Laryngoscope Investigative Otolaryngology 2019;4 (1):116-23. (PMID: 30828628)

2. Kim JS, Zee DS. Benign paroxysmal positional vertigo. New England Journal of Medicine 2014;370 (12):1138-47. (PMID: 24645946)

3. Bhattacharyya N, Baugh RF, Orvidas L, Barrs D, Bronston LJ, Cass S, et al. Clinical practice guideline: benign paroxysmal positional vertigo. Otolaryngology--Head and Neck Surgery 2008;139 (5 suppl): 47 81. (PMID: 18973840)

4. Gaur S, Awasthi SK, Bhadouriya SKS, et al. Efficacy of Epley's maneuver in treating BPPV patients: a prospective observational study. International journal of otolaryngology 2015; 2015: 487160. (PMID: 26495002)

5. Von Brevern M, Radtke A, Lezius F, et al. Epidemiology of benign paroxysmal positional vertigo: a population based study. Journal of Neurology, Neurosurgery \& Psychiatry 2007;78 (7): 710-5. (PMID: 17135456)

6. Kollén L, Frändin K, Möller M, et al. Benign paroxysmal positional vertigo is a common cause of dizziness and unsteadiness in a large population of 75-year-olds. Aging clinical and experimental research 2012; 24 (4): 317-23. (PMID: 23238307)

7. Balatsouras D, Koukoutsis G, Fassolis A, et al. Benign paroxysmal positional vertigo in the elderly: current insights. Clinical interventions in aging 2018; 13: 2251-2266. (PMID: 30464434)

8. Fujimoto C, Kawahara T, Kinoshita M, et al. Aging is a risk factor for utricular dysfunction in idiopathic benign paroxysmal positional vertigo. Frontiers in neurology 2018; 9: 1049. (PMID: 30559714)

9. Chua K, Gans R, Spinks S. Demographic and clinical characteristics of BPPV patients: a retrospective large cohort study of 1599 patients. Journal of Otolaryngology-ENT Research 2020;12(1):20-30. (DOI:10.15406/joentr.2020.12.00451).
10. Moreno NS, André APdR. Number of maneuvers need to get a negative Dix-Hallpike test. Brazilian Journal of Otorhinolaryngology 2009; 75 (5): 650-3. (PMID: 19893930).

11. Korkmaz M, Korkmaz H. Cases requiring increased number of repositioning maneuvers in benign paroxysmal positional vertigo. Brazilian Journal of Otorhinolaryngology 2016;82 (4): 452-7. (PMID: 26614043)

12. Santos MA, Bittar R. Vertigo and metabolic disorders. The International Tinnitus Journal 2012; 17 (1): 16-20. (PMID: 23906822)

13. Batuecas-Caletrio A, Trinidad-Ruiz G, Zschaeck C, et al. Benign paroxysmal positional vertigo in the elderly. Gerontology. 2013; 59 (5): 408-12. (PMID: 23689314)

14. Luryi AL, Lawrence J, LaRouere M, et al. Treatment of patients with benign paroxysmal positional vertigo and severe immobility using the particle repositioning chair: a retrospective cohort study. Annals of Otology, Rhinology \& Laryngology 2018; 127 (6): 390-4. (PMID: 29732909)

15. Korres S, Balatsouras D, Ferekidis E. Prognosis of patients with benign paroxysmal positional vertigo treated with repositioning manoeuvres. The Journal of laryngology and otology 2006; 120( 7): 528. (PMID: 16556351)

16. Hornibrook J. Benign paroxysmal positional vertigo (BPPV): history, pathophysiology, office treatment and future directions. International journal of otolaryngology 2011; 2011: 835671. (PMID: 21808648)

17. Parham K. Benign paroxysmal positional vertigo: an integrated perspective. Advances in Otolaryngology 2014. (DOI: 10.1155/2014/792635)

18. Vaduva C, Esteban-Sanchez, J., Sanz-Fernandez, R., et al. Prevalence and management of post-BPPV residual symptoms. European Archives of Oto-Rhino-Laryngology 2018; 275: 1429-37. (PMID: 29687182)

19. Mizukoshi K, Watanabe Y, Shojaku H, et al. Epidemiological studies on benign paroxysmal positional vertigo in Japan. Acta Oto-Laryngologica 1988; 105 (sup447): 67-72. (PMID: 3188895) 
20. Ferrari S, Monzani D, Baraldi S, et al. Vertigo "in the pink": the impact of female gender on psychiatric-psychosomatic comorbidity in benign paroxysmal positional vertigo patients. Psychosomatics 2014; 55 (3): 280-8. (PMID: 23756120)

21. Wang J, Chi F-I, Jia X-h, et al. Does benign paroxysmal positional vertigo explain age and gender variation in patients with vertigo by mechanical assistance maneuvers? Neurological Sciences 2014; 35 (11): 1731-6. (PMID: 24844790).

22. Choi HG, Lee JK, Kong IG, et al. Osteoporosis increases the risk of benign paroxysmal positional ver- tigo: a nested case-control study using a national sample cohort. European Archives of Oto-Rhino-Laryngology 2019; 276 (2): 335-42. (PMID: 30511104).

23. Ogun OA, Büki B, Cohn ES, et al. Menopause and benign paroxysmal positional vertigo. Menopause 2014; 21 (8): 886-9. (PMID: 24496089)

24. Li M, Tomlinson G, Naglie G, et al. Geriatric comorbidities, such as falls, confer an independent mortality risk to elderly dialysis patients. Nephrology Dialysis Transplantation 2007; 23 (4): 1396-400. (PMID: 18057068) 